\title{
The effect of FCYRIIA and FCYRIIB on coronary artery lesion formation and intravenous immunoglobulin treatment responses in children with Kawasaki disease
}

\author{
Ling-Sai Chang1,2,3, Mao-Hung Lo ${ }^{1,3}$, Sung-Chou $\mathrm{Li}^{4}$, Ming-Yu Yang ${ }^{2,5}$, Kai-Sheng \\ Hsieh $^{1,3}$ and Ho-Chang Kuo ${ }^{1,3}$ \\ ${ }^{1}$ Kawasaki Disease Center, Kaohsiung Chang Gung Memorial Hospital, Kaohsiung, Taiwan \\ ${ }^{2}$ Graduate Institute of Clinical Medical Sciences, College of Medicine, Chang Gung University, Kaohsiung, Taiwan \\ 3 Department of Pediatrics, Kaohsiung Chang Gung Memorial Hospital and Chang Gung University College of Medicine, \\ Kaohsiung, Taiwan \\ ${ }^{4}$ Genomics and Proteomics Core Laboratory, Department of Medical Research, Kaohsiung Chang Gung Memorial Hospital and \\ Chang Gung University College of Medicine, Kaohsiung, Taiwan \\ ${ }^{5}$ Department of Otolaryngology, Kaohsiung Chang Gung Memorial Hospital and Chang Gung University College of Medicine, \\ Kaohsiung, Taiwan \\ Correspondence to: Ming-Yu Yang, email: yangmy@gmail.com
}

Kai-Sheng Hsieh, email: kshsieh@adm.cgmh.org.tw

Ho-Chang Kuo, email: erickuo48@yahoo.com.tw

Keywords: coronary artery lesions; FcyRIIA; FcyRIIB; intravenous immunoglobulin; Kawasaki disease; Immunology and Microbiology Section; Immune response; Immunity

Received: July 15, $2016 \quad$ Accepted: November 14, $2016 \quad$ Published: November 21, 2016

\section{ABSTRACT}

Previous research has found patients with the FCYRIIIB NA1 variant having increased risk of intravenous immunoglobulin (IVIG) resistance in Kawasaki disease (KD). Our previous studies revealed that elevated FcyRIIA expression correlated with the susceptibility of KD patients. We conducted this research to determine whether and how Fcy receptors affect the susceptibility, IVIG treatment response, and coronary artery lesions (CAL) of KD patients. The activating FCYRIIA and inhibitory FCYRIIB methylation levels of seven patients with KD and four control subjects were examined using HumanMethylation27 BeadChip. We enrolled a total of $44 \mathrm{KD}$ patients and 10 control subjects with fevers. We performed real-time RT-PCR to determine the FCYRIIA and FCYRIIB expression levels, as well as a luciferase assay of FCYRIIA. We found a considerable increase in methylation of both FCYRIIA and FCYRIIB in KD patients undergoing IVIG treatment. Promoter methylation of FcYRIIA inhibited reporter activity in $\mathrm{K} 562$ cells using luciferase assay. The FCYRIIB mRNA expression levels were not found to increase susceptibility, CAL formation, or IVIG resistance. FCYRIIA mRNA expression levels were significantly higher in IVIG-resistant patients than in those that responded to IVIG during the pre-treatment period. Furthermore, the FCYRIIA/IIB mRNA expression ratio was considerably higher in KD patients with CAL than in those without CAL. FCYRIIA and FCYRIIB both demonstrated increased methylation levels in KD patients that underwent IVIG treatment. FcyRIIA expression influenced the IVIG treatment response of KD patients. The FCYRIIA/IIB mRNA expression ratio was greater in KD patients with CAL formation.

\section{INTRODUCTION}

A type of vasculitis, Kawasaki disease (KD) occurs most commonly in children, surpassing acute rheumatic fever as their leading cause of acquired heart disease [1]. KD is an acute multi-system vasculitis syndrome that consists of a prolonged fever and at least four of the following five diagnostic criteria: polymorphous rash, 
Table 1: Demographic data of Kawasaki disease patients and control subjects

\begin{tabular}{|c|c|c|c|}
\hline & Controls & Kawasaki disease & $p$-value \\
\hline mRNA study & $(N=10)$ & $(N=44)$ & \\
\hline Age (year) & $1.95 \pm 0.84$ & $1.52 \pm 0.17$ & 0.268 \\
\hline Male & $6(60 \%)$ & $21(47.8 \%)$ & 0.484 \\
\hline Clinical data & $\begin{array}{l}\text { Upper respiratory tract infection }(N=2) \\
\text { Lower respiratory tract infection }(N=6) \\
\text { Gastroenteritis }(N=2)\end{array}$ & $\begin{array}{l}\text { IVIG resistance: } 4 \text { patients }(9 \%) \\
\text { CAL: } 7 \text { patients }(15.9 \%)\end{array}$ & \\
\hline Pyrosequencing & $(N=54)$ & $(N=42)$ & \\
\hline Age $<5$ years & $100 \%$ & $100 \%$ & \\
\hline Male & $31(57.4 \%)$ & $27(64.3 \%)$ & 0.534 \\
\hline
\end{tabular}

a) Data are shown as mean \pm standard error.

b) Abbreviations: CAL, coronary artery lesions; IVIG, intravenous immunoglobulin

non-exudative conjunctivitis, oral-mucosal involvement, extremity desquamation, and cervical lymphadenopathy. The immunopathogenesis of KD is complex and not yet completely understood. Immune complexes are occasionally found in the peripheral blood of KD patients [2]. Genetic mapping and candidate gene analyses have indicated that $\mathrm{KD}$ is correlated with a variety of chromosomal regions, as well as several candidate genes that control susceptibility of KD, coronary aneurysm formation, and intravenous immunoglobulin (IVIG) treatment responsiveness [3]. A diagnosis of Kawasaki disease must be considered for any child with a prolonged fever of unknown origin. For KD treatment, a single high dose of IVIG is highly effective for lowering both the fever and the incidence of coronary aneurysms. However, IVIG's pharmacological mechanisms are also not fully understood. Treatments have been proposed based on the immune suppressive action of IVIG, such as stimulating the upregulation of inhibitory Fc $\gamma$ receptors on macrophages and competing with immunocomplexes to activate $\mathrm{Fc} \gamma$ receptors [4]. In a study of mice with inflammatory arthritis, IVIG with glycans terminating in $\alpha 2,6$ sialic acids attached to the lectin dendritic-cellspecific ICAM-3 grabbing non-integrin (DC-SIGN) and promoted the creation of IL-33, which then expanded the IL-4+ basophils. These Th2 cytokines can inhibit autoantibody-mediated inflammation by modulating Fc $\gamma$ RIIB expression on effector cells [5]. A number of Fc $\gamma$ functions are maintained among various species. In humans, IVIG treatment inhibits dendritic cell function through the Th2 cytokine-mediated (IL-4 and IL-13) downregulation of $F c \gamma R I I A$ and $I F N-\gamma R 2$, not through the upregulation of $F c \gamma R I I B$ [6]. Th2 cells are critical for host protection against multicellular parasites, such as intestinal helminthes; when such cells are deregulated, they can contribute to such atopic diseases as asthma and enhance forms of vasculitis like eosinophilic granulomatosis with polyangiitis and inflammatory arthritis [5, 7]. Previous research has indicated that $\mathrm{Th} 2$ cells are crucial in KD's pathogenesis. Elevated eosinophil levels after IVIG treatment may be correlated with IVIG responsiveness [8].
We have demonstrated that Th2 immune-related responses (eosinophils, IL-4, IL-5, eotaxin, and eosinophil cationic protein) correlated with the susceptibility to $\mathrm{KD}$ and disease outcomes, as well as that interleukin-31, which is known to be related to Th2 cytokines, correlated with coronary artery lesions (CAL) when compared to the febrile control subjects $[9,10]$. Genome-wide association studies and linkage analyses of KD have found that genes that contributed to eosinophil degranulation (a functional polymorphism in the $\mathrm{IgG}$ receptor gene $\left.F_{c} \gamma R I I A\right)$ correlated with KD [11]. Furthermore, an increased risk for atopic dermatitis was found in preschool children with KD in Taiwan [12]. We also found a positive correlation between $\mathrm{KD}$ and the risk of allergic diseases, which agrees with the findings of previous research [13].

Specific $F c \gamma R$ polymorphisms have been determined to be associated with the induction or severity of KD and patient responsiveness to IVIG [14]. However, each of these genetic associations is limited by the unequal polymorphic variations among the various ethnic groups studied. The Fc $\gamma$ RIIA-H131 variant correlates with KD, while KD patients' responsiveness to IVIG therapy is strongly associated with the $F_{C} \gamma R I I I B$ genotype; the NA1 variant significantly reduces the probability of a proper clinical outcome [15]. Likewise, the lower copy number of Fc $R$ RIIC has been correlated with KD susceptibility [16].

These results indicate that $\mathrm{F} c \gamma$ receptors may be related to KD's clinical and pathological features; however, not much is known about their functional relationship. To better understand this phenomenon, we measured Fc $\gamma$ receptors' (activating Fc $F_{R I I A}$ and inhibitory $F_{c \gamma}$ RIIB) mRNA levels in KD patients and investigated the clinical importance of Fc $\gamma$ receptors. Previous studies have found that epigenetic regulatory mechanisms correlate with a predisposition to KD and IVIG resistance [17]. The current study researched the DNA methylation of Fc $\gamma$ RIIA and $F_{c} \gamma R I I B$ using HumanMethylation27 BeadChip (Illumina, San Diego, CA, USA) in KD patients. A pyrosequencing assay was used to carry out confirmation with another cohort of DNA methylation array. We further evaluated 
the functional properties of the $F c \gamma R I I A$ promoter $\mathrm{CpG}$ methylation using a luciferase assay.

\section{RESULTS}

\section{Demographic data}

We recruited a total of 44 patients with Kawasaki disease ( $1.52 \pm 0.17$ years old, 21 male) for this casecontrol study (Table 1). Another 10 patients with an acute febrile infectious disease ( $1.95 \pm 0.84$ years old, 6 male $)$ were selected as control subjects. The acute infections among the control group were upper or lower respiratory tract infections or gastroenteritis. No significant difference in age or gender was found between the KD patients and the control group. Of all the participants, seven patients $(15.9 \%)$ had CAL formation, and four patients $(9 \%)$ were IVIG resistant.

\section{FcyRIIA and FcyRIIB expression and CAL formation}

In the expression analysis, our data is presented as normalized to the mRNA levels of the control group

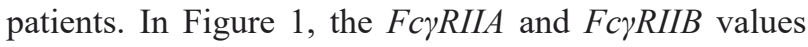
are shown normalized to the febrile controls. In our previous research, an increase in FcyRIIA mRNA levels was discovered in KD patients [17], but we found no significant differences between the $\mathrm{KD}$ patients and the febrile controls with regard to the mRNA levels of $F c \gamma R I I B$ in this study. In a previous report, we observed a considerable decrease in Fc $R I I A$ mRNA expression after KD patients received IVIG treatment [17]. During the study period, $F c \gamma R I I B$ expression gradually reduced after IVIG treatment, although it was not statistically significant. No significant differences were found with

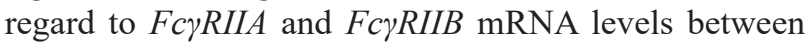
KD patients with or without CAL before and after IVIG therapy. The FcyRIIA expression varied considerably between the KD patients that responded to IVIG treatment and those that were IVIG resistant ( $p=0.025$ ) (Figure 2). On the other hand, we observed no significant difference between the mRNA levels of $F c \gamma R I I B$ the IVIG-resistant patients and the IVIG-responsive patients.

Compared to the febrile controls, KD patients demonstrated a considerably increased FcyRIIA/IIB mRNA ratio $(13.29 \pm 3.72$ vs. $1.01 \pm 0.14 ; P<0.001)$. The FCyRIIA/IIB mRNA expression ratio was also significantly higher in patients with CAL when compared to those without CAL ( $22.22 \pm 8.56$ vs. $11.60 \pm 4.10 ; p=0.007)$ (Figure 3). These findings indicate a shifting balance of the two opposing $F c \gamma R$ isoforms toward an activating phenotype in KD patients and patients with CAL.

\section{Methylation levels of $F c \gamma R I I A$ and $F c \gamma R I I B$}

Previous evidence has indicated that epigenetic changes may offer an alternate explanation of KD's pathophysiology. We performed additional analysis of methylation with the Illumina HumanMethylation27

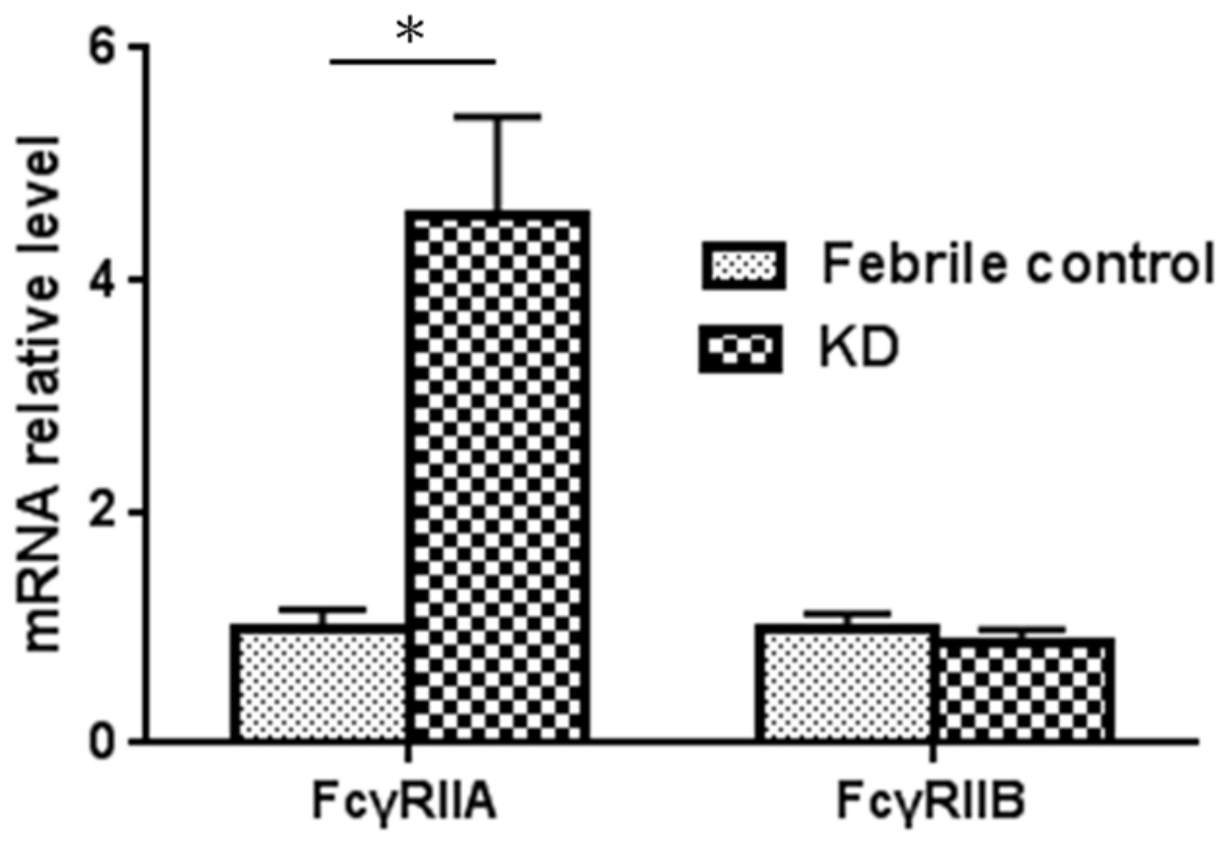

Figure 1: $F c \gamma R I I A$ and $F c \gamma R I I B$ mRNA expression in KD patients and control subjects (KD patients, $N=44$; control, $N=10)$. *A $p$-value $<0.05$ between the two groups. 
Table 2: Significant increase in methylation of $F c \gamma R I I A$ and $F c \gamma R I I B$ after intravenous immunoglobulin treatment

\begin{tabular}{|c|c|c|c|c|c|c|}
\hline Target ID & Symbol & $\begin{array}{c}\mathrm{FC} \\
N=4 \\
\end{array}$ & $\begin{array}{l}\text { KD1 } \\
N=7 \\
\end{array}$ & $\begin{array}{l}\text { KD-3 } \\
N=7 \\
\end{array}$ & $\begin{array}{c}p \text {-value } \\
\text { (KD1 vs. FC) }\end{array}$ & $\begin{array}{c}p \text {-value } \\
\text { (KD1 vs. KD3) }\end{array}$ \\
\hline $\operatorname{cg} 22436411$ & Fc $\gamma$ RIIB & $0.070 \pm 0.043$ & $0.062 \pm 0.041$ & $0.132 \pm 0.063$ & 0.957 & $0.013^{*}$ \\
\hline cg27470554 & Fc $\gamma$ RIIA & $0.052 \pm 0.013$ & $0.046 \pm 0.015$ & $0.072 \pm 0.011$ & 0.387 & $0.002 * *$ \\
\hline $\operatorname{cg} 24422489$ & $\overline{\text { Fc } \gamma \text { RIIA }}$ & $0.620 \pm 0.127$ & $0.465 \pm 0.167$ & $0.727 \pm 0.129$ & $0.007 *$ & $0.002 * *$ \\
\hline
\end{tabular}

a) Data are shown as mean \pm standard deviation.

b) We tested p-values using the independent sample $t$-test between FC and KD1; we tested p-values using the paired $t$-test between KD1 and KD3.

c) $*$ indicating $\mathrm{p}$-value $<0.05$. ; ** indicating $p$-value $<0.005$.

d) Abbreviations: FC, febrile control; KD1, Kawasaki disease before intravenous immunoglobulin; KD3, at least three weeks after intravenous immunoglobulin.

BeadChip array in order to explore the methylation profiles of $F c \gamma R I I A$ and $F c \gamma R I I B$ that could account for the disease's clinical pathophysiology. We recruited seven children with KD, as well as four febrile children as controls, for this study. We carried out microarray analysis with the HumanMethylation27 BeadChip on genomic DNA from the entire blood, finding significant hypomethylation of the $F_{C} \gamma R I I A(\operatorname{cg} 24422489)$ promoters, which are functionally associated with genes discovered by prior studies to be differentially methylated in $\mathrm{KD}$ patients [17]. In this study, we found that FcyRIIA (cg27470554) and FcrRIIB (cg22436411) methylation levels were not lower in KD patients than in the febrile controls ( $p=0.387$ and $p=0.957$, respectively) (Table 2). HumanMethylation27 FcyRIIA (cg27470554 and cg24422489, $p=0.002$ and 0.002 respectively) and Fc $\gamma$ RIIB (cg22436411, $p=0.013)$ demonstrates noticeably higher methylation levels after patients underwent IVIG treatment (Table 2). To verify the methylation array results, we utilized target-specific sequencing at $\operatorname{cg} 22436411$ in the $F_{c \gamma} R I I B$ promoter region through pyrosequencing, which resulted in only one methylation site. With an additional $42 \mathrm{KD}$ patients and 54 controls, the methylation corroboration showed that this Fc $\gamma$ RIIB hypomethylation difference did not achieve statistical significance.

\section{Functional analysis of the $F c \gamma R I I A$ promoter}

Using both the HumanMethylation27 BeadChip array and pyrosequencing methods, we observed $F_{C} \gamma$ RIIA promoter hypomethylation in KD patients. Due to the luciferase assay, we decided to research whether DNA methylation could regulate $F c \gamma R I I A$ expression instead of using $\mathrm{CpG}$ sites mutation [17]. To establish a direct link between the FcrRIIA expression levels and their methylation state, we performed reporter gene analysis that included cloning the Fc $\gamma$ RIIA promoter region.

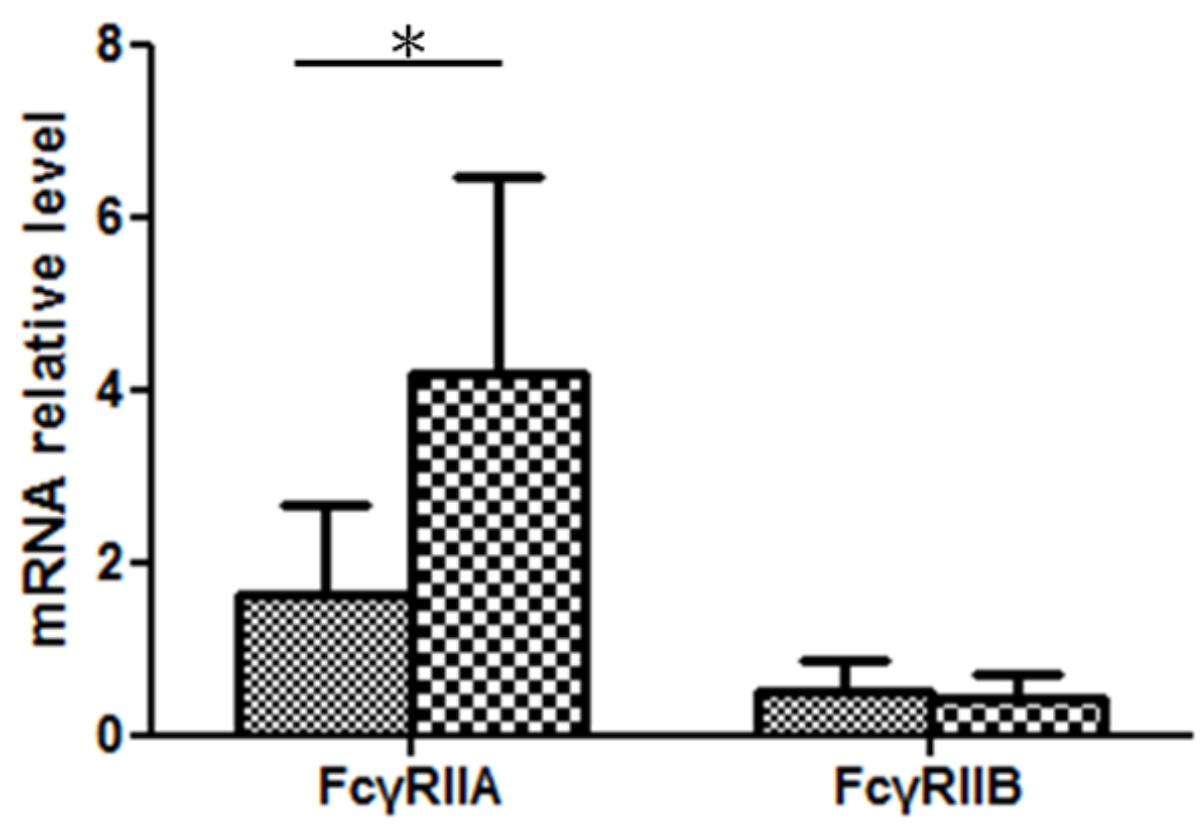

$\mathbf{m}$ IVIG sensitive
$\mathbf{\infty}$ IVIG resistant

Figure 2: Considerably higher $F c \gamma R I I A$ mRNA levels in IVIG-resistant KD patients (IVIG resistant $N=4$; IVIG sensitive, $N=40$ ) 
The CpG methylase Sss I-treated and untreated plasmids were transfected into K562 cells. As shown in Figure 4, the results from the luciferase experiments, which were repeated at least six times, demonstrate that promoter activity was distinctly influenced by $\mathrm{CpG}$ methylation. These findings indicate that both of the $\mathrm{CpG}$ containing reporter vectors were successfully inhibited through DNA methylation by $\mathrm{CpG}$ methylase Sss I. To determine whether partial methylation may also influence reporter activity, the plasmids were either methylated using site-specific methylases Hha I, and/or Hpa II or were left unmethylated. Luciferase activity was assayed in K562 cells once transient transfection was completed. Figure 4 shows that the reporter activity of the differentially methylated plasmids significantly decreased.

\section{DISCUSSION}

The current study has found that treating KD patients with IVIG can considerably reduce the elevated mRNA levels of FcyRIIA. Our previous research validated $F_{C} \gamma R I I A$ methylation [17]. The present results which find $F C \gamma R I I A$ expression to be significantly higher in IVIG-resistant patients support our previous study that shows that KD with IVIG resistance demonstrated hypomethylation [17]. Furthermore, we recently discovered a significant correlation among the promoter methylation of $F c \gamma R I I A$, susceptibility of Kawasaki disease, and therapeutic outcomes of IVIG treatment [18]. Through functional testing, we discovered that in vitro methylation of the Fc $\gamma$ RIIA promoter decreased luciferase

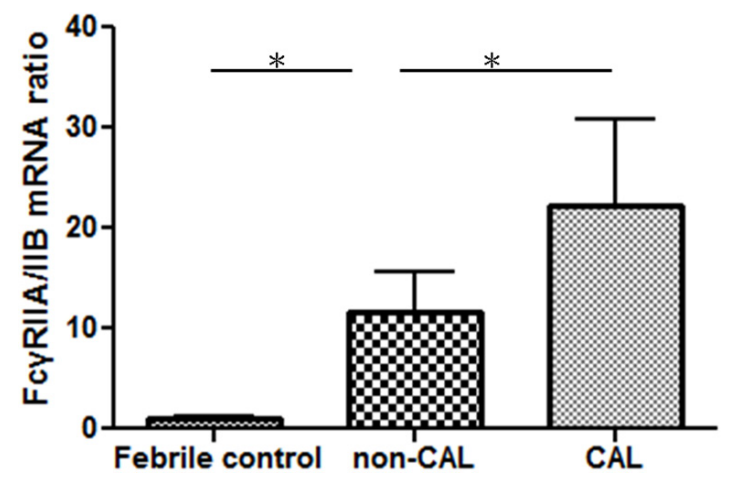

Figure 3: The $F c \gamma R I I A$ and $F c \gamma R I I B$ mRNA expression ratio of KD patients with $(N=7)$ and without $\mathrm{CAL}(N=37)$ and the control subjects $(N=10)$ was determined using quantitative PCR. ${ }^{*} p$-value $<0.05$.

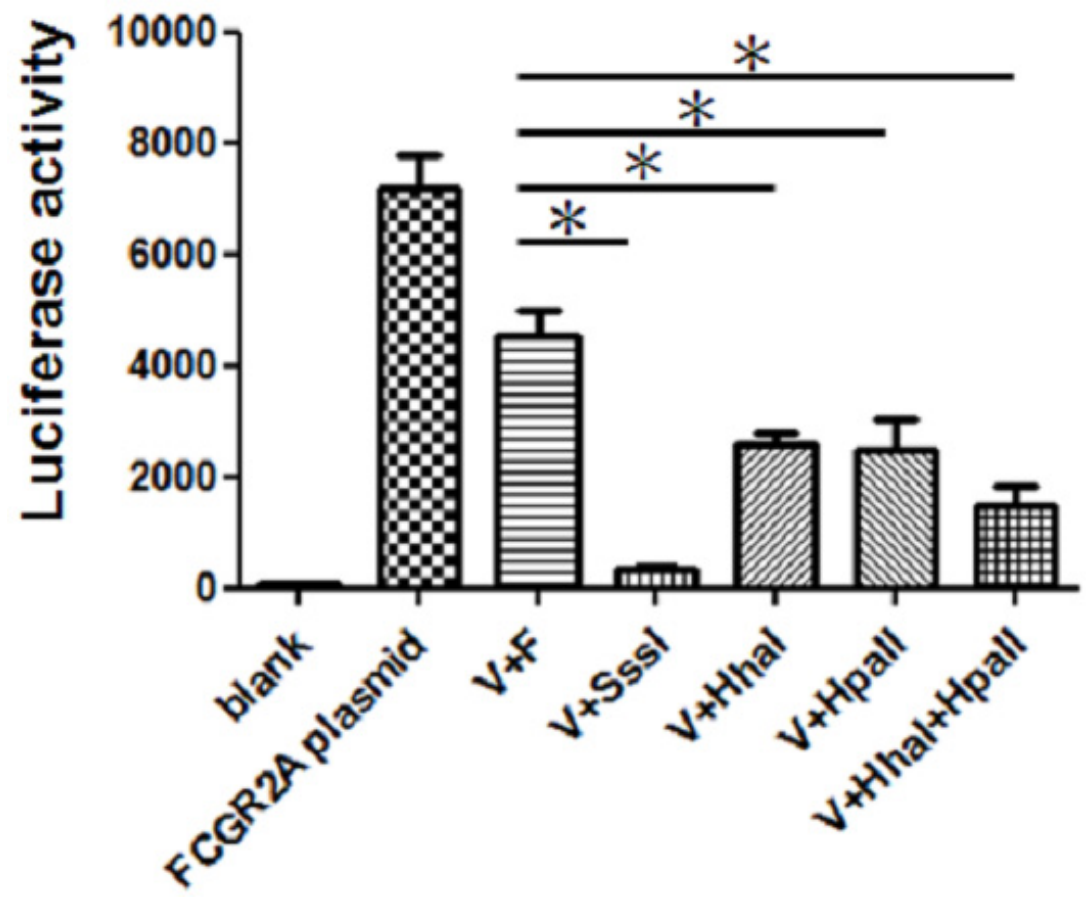

Figure 4: Comparative analysis of differential promoter methylation. In vitro methylation inhibits $F c \gamma R I I A$ promoter reporter activity in K562 cells. $(* p<0.05)$. All data are representative of at least six experiments. The transfection of K562 cells was performed with LightSwitch vector and $F c \gamma R I I A$ promoter plasmid $(\mathrm{V}+\mathrm{F})$ or vector $(\mathrm{V})$ treated with methyltransferase SssI, Hha I, or Hpa II methylases. 
activity. Elevated $\mathrm{F} c \gamma R I I A / F c \gamma R I I B$ expression has also been seen in patients with CAL; therefore, the ratio is altered to favor cell activation.

Human Fc gamma receptors (Fc $\gamma \mathrm{Rs}$ ), which are the receptors for the Fc region of immunoglobulinG (IgG) antibodies, are vital in this process as they control the production of tissue- and pathogen-specific cytokines [19]. Fc $\gamma$ Rs are responsible for such processes as uptake, antigen presentation, and antibody dependent cellular cytotoxicity [19]. Inhibitory FcyRIIB receptors are primarily expressed by B cells, monocytes, and macrophages and are not probable factors to explain IVIG's immunomodulatory capacity [20]. FcyRIIB influences humans' susceptibility to several autoimmune diseases, including systemic lupus erythematosus, rheumatoid arthritis, anti-GBM disease, and idiopathic thrombocytopenic purpura [21]. Genes have functional polymorphisms that can manage these receptors to alter the balance between activation and inhibition and ultimately their interaction. Elevated Fc $\gamma$ RIIA/IIB mRNA expression levels in untreated KD patients reflect the potential role of a disrupted Fc $\gamma R$ balance in the pathogenesis of CAL formation. Such expression variations may, at least in part, occur secondary to inflammation. IVIG responses in KD patients depend on $F_{c} \gamma R I I B$ polymorphisms in a racially dependent manner [22]. The Asian individuals studied lacked these SNPs, which agrees with our reports that found that the $F c \gamma R I I B$ mRNA expression levels did not appear to affect KD susceptibility, CAL formation, or IVIG resistance. We performed independent validation of methylation profiles by analyzing $F c \gamma R I I B$ data from clinical samples of patients' blood. Pyrosequencing is superior to methylation-specific PCR due to such advantages as requiring only a small amount of DNA, flexibility, accuracy, and quantification [23].

Various studies have confirmed a significant relationship between $F c \gamma R I I A$ and KD. Elevated Fc $\gamma$ RIIA expression has been observed in KD patients [17], and the epigenetic dysregulation of $F c \gamma R I I A$ is vital for facilitating the disease's clinical outcome [18]. Furthermore, IVIG treatment can potentially alter DNA methylation/ demethylation dynamics. This data shows that IVIG treatment can increase the DNA methylation of Fc $\gamma$ RIIA and $F_{C} \gamma R I I B$. As for the functional significance of $F_{C} \gamma R I I A$ methylation, which we determined by testing the influence of promoter methylation in vitro, our experimental data clearly shows that methylation of the $F c \gamma R I I A$ promoter can control luciferase activity in K562 cells.

As described in our previous study of Fc $\gamma$ RIIA mRNA and KD, functional studies have been performed using CpG sites' point mutation with pGL3-Basic vector in cultured Chang liver cells [17]. Unlike the present study, that study focused specifically on the regional $\mathrm{CpG}$ site of cg24422489 instead of the whole promoter. In this study, luciferase assay with reporter vector (LightSwitch) in K562 cells also revealed that methylation of the
Fc $\gamma$ RIIA promoter using methyltransferase significantly decreased promoter activity. However, methylation cannot be precisely targeted to individual single CpGs as site-specific intervention using this method. The findings of both of the functional luciferase studies strongly suggest that methylation of the Fc $\gamma$ RIIA promoter could significantly reduce gene expression.

This study has some limitations. First, we did not

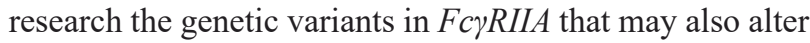
the variability of the $F c \gamma R I I A$ expression. We also did not assess other expression levels of $\mathrm{Fc} \gamma \mathrm{R}$ or the expression levels of different blood cells.

The present study's results confirm that $F_{c} \gamma R I I A$ mRNA was upregulated in KD patients with IVIG resistance. We also determined that DNA methylation in the $F c \gamma R I I A$ promoter region was the fundamental mechanism for the expression differences of Fc $\gamma$ RIIA in $\mathrm{KD}$, which was verified through in vitro cell culture experiments. Furthermore, our data indicates that $F_{C} \gamma R I I A / F_{c} \gamma R I I B$ expression is elevated in patients with CAL. Therefore, $F c \gamma R I I A$ can be considered a valuable marker for predicting KD's treatment outcome. Our discovery that Fc $\gamma$ RIIA methylation influences epigenetic regulators reinforces the importance of such changes in KD's pathophysiology and emphasizes the potential of epigenetic modulators for successfully treating KD.

\section{MATERIALS AND METHODS}

\section{Subjects}

This study features 44 patients diagnosed with KD, as well as 10 febrile, age- and gender-matched control subjects. We took peripheral blood samples at three points during the study: before IVIG treatment (KD1), shortly after IVIG treatment (within three days, KD2), and at least three weeks after IVIG treatment (KD3), and then analyzed $F c \gamma R I I A$ and $F c \gamma R I I B$ mRNA levels using a quantitative real-time polymerase chain reaction. Subjects were diagnosed with KD by a clinician using the recommended universal KD criteria proposed by the American Heart Association (AHA) [24]. KD was diagnosed in any child with a fever that lasts longer than five days and manifested four of the following five criteria: diffuse mucosal inflammation with strawberry tongue and fissure lips, bilateral nonpurulent conjunctivitis, indurative angioedema of the hands and feet, dysmorphic skin rashes, and unilateral cervical lymphadenopathy [25]. Pursuant to the guidelines of the American Academy of Pediatrics and the American Heart Association, KD patients were treated with high doses of IVIG $(2 \mathrm{~g} / \mathrm{kg})$ within $12 \mathrm{~h}$ of diagnosis [24]. IVIG responsiveness (IVIG sensitivity) is defined as fever reduction within 48 hours after initial IVIG treatment and no reoccurrence of fever 
$\left(>38^{\circ} \mathrm{C}\right)$ for seven or more days. We used two-dimensional echocardiography to identify the presence of CAL, which are defined as coronary arteries with a diameter larger than $3 \mathrm{~mm}$ (or $4 \mathrm{~mm}$ in patients older than five years). Among the participants, seven developed CAL. This study was approved by Chang Gung Memorial Hospital's Internal Review Board, and we obtained written informed consent from the parents or guardians of all the participating children.

\section{Confirming DNA methylation array using pyrosequencing assay}

In our previous studies, we carried out DNA methylation array using the HumanMethylation27 BeadChip and then pyrosequencing assay to confirm the DNA methylation array. A total of $500 \mathrm{ng}$ genomic DNA was treated with bisulfite using an EZ DNA methylation kit (ZYMO Research) in accordance with the manufacturer's instructions. It was then washed in $20 \mu \mathrm{l}$ of Tris-Buffer (10 $\mathrm{mM})$. All primers were specifically designed for bisulfiteconverted DNA, which was amplified by PCR using unbiased nested primers. PCR was performed in a $25-\mu \mathrm{l}$ reaction mixture of $25 \mathrm{ng}$ of bisulfite-converted DNA, 1X Pyromark PCR Master Mix (Qiagen, Valencia, CA,

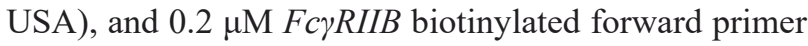
5'-ATGTTGAGGGTGAATAAATGG-3' and reverse primer 5'-biotin-ATATTCCCCAAAAAATAAATTACCC CTAAC-3' with the following PCR program: $95^{\circ} \mathrm{C}$ for 5 min, then 45 cycles of $95^{\circ} \mathrm{C}$ for $30 \mathrm{~s}$, followed by $55^{\circ} \mathrm{C}$ for $30 \mathrm{~s}$, and $72^{\circ} \mathrm{C}$ for $30 \mathrm{~s}$, with a final extension of $72^{\circ} \mathrm{C}$ for $5 \mathrm{~min}$. After amplification, the biotinylated PCR products were purified and incubated with the sequencing primer 5'-GGTAATGAGGATGATGATA-3', which was created to be attached to the $\mathrm{CpG}$ sites of interest. We evaluated DNA methylation with quantitative pyrosequencing using a PyroMark Q24 instrument (Qiagen, Valencia, CA, USA) and established the DNA methylation percentage at each CpG site using PyroMark Q24 1.010 software.

\section{Real-time RT-PCR of $F c \gamma R I I A$ and $F c \gamma R I I B$}

All RNA was isolated from white blood cells using the FavorPrep Blood/Cultured Cell Total RNA Purification Kit (Favorgen, cat. No. FABRK001-1). We utilized the high-performance reverse-transcriptase system (EPICENTRE) to perform reverse transcription of the RNA samples. Both $F c \gamma R I I A$ and $F_{c} \gamma R I I B$ expression levels were detected with real-time RT-PCR using the SYBR Green PCR Master Mix and ABI Prism 7500 Sequence Detection System (Applied Biosystems). The primers used to amplify $F c \gamma R I I A$ mRNA were 5'-TCA TTG TGG CTG TGG TCA TT G-3'(forward) and 5'CCT GGG GTT CAG AGT CAT GT-3'(reverse); those for amplifying $F c \gamma R I I B$ mRNA were 5'GTT GGG GCT GAG AAC ACA AT 3' (forward) and 5'ACA GGG AGC TTC AGG ACT CA 3' (reverse).

\section{Luciferase assay to functionally analyze FcyRIIA promoter CpG methylation}

To investigate $F c \gamma R I I A$ promoter activity, the human $F c \gamma R I I A$ promoter-reporter luciferase construct commercially synthesized by LightSwitch was processed using the restriction enzymes Mlu I and Bgl II; said digestion process was then confirmed with agarose gel ectrophoresis. We treated 5 ug plasmids with nonspecific $\mathrm{CpG}$ methyltransferase SssI (New England BioLabs), Hha I, Hpa II methylases, and Hha I + Hpa II for 4 hours in accordance with the manufacturer's instructions, and the change of $\mathrm{CpG}$ was analyzed with plasmid DNA bisulfite modification and pyrosequencing using specific primers (Pyromark ID96 system, Biotage). The methylated plasmids were ligated into the luciferase vector. The aforementioned inserted fragments and genes were confirmed using DNA sequencing. The human immortalized myelogenous leukemia cell line K562 was co-transfected with the methylated human Fc $\gamma$ RIIA promoter-reporter luciferase construct in accordance with the manufacturer's instructions. The transfection of the plasmids in K562 cells was performed with Lipofectamine 2000 (Invitrogen). Once transfected for 24 hours, promoter-reporter luciferase activity was assessed using a Dual-Luciferase Reporter Assay System (Promega). We used the unmethylated human $F c \gamma R I I A$ promoter-reporter luciferase construct as the control.

\section{Statistics}

The data in this study are expressed as mean \pm standard error. We used student's t-test to compare demographic data and the Mann-Whitney U-test to compare mRNA expression levels. Any differences in the blood's measured variables before and after IVIG treatment were pairwise analyzed using the Friedman test. A p-value less than 0.05 was considered statistically significant. All statistical tests were carried out with SPSS 14.0 for Windows XP (SPSS, Inc., Chicago, IL, USA).

\section{ACKNOWLEDGMENTS}

We would like to thank the Genomics \& Proteomics Core Laboratory of the Department of Medical Research at Kaohsiung Chang Gung Memorial Hospital for its technical support. 


\section{CONFLICTS OF INTEREST}

The authors hereby declare that they have no conflicts of interest to disclose with regard to this article.

\section{GRANT SUPPORT}

This study received support from grants provided by the Ministry of Science and Technology (MOST: 105-2314-B-182 -050 -MY3), the Ministry of Health and Welfare (PMRPG8E0011), and Chang Gung Memorial Hospital (CMRPG8C1082, CMRPG8B0212, CMRPG8D1562, CORPG8F0011, CMRPG8E1611 and CMRPG8D0521) in Taiwan.

\section{REFERENCES}

1. Kuo HC, Yang KD, Chang WC, Ger LP, Hsieh KS. Kawasaki disease: an update on diagnosis and treatment. Pediatr Neonatol.2012;53(1):4-11.

2. Mason WH, Jordan SC, Sakai R, Takahashi M, Bernstein B. Circulating immune complexes in Kawasaki syndrome. Pediatr Infect Dis. 1985;4(1):48-51.

3. AH. R. Kawasaki disease: novel insights into etiology and genetic susceptibility. Annu Rev Med 2011;62:69-77.

4. Chong BH CJ. IVIg immune inhibitory activity: APC is key. Blood. 2010;Mar 4;115(9):1663-1664.

5. Anthony RM KT, Wermeling F, Ravetch JV. Intravenous gammaglobulin suppresses inflammation through a novel T(H)2 pathway. Nature. 2011;Jun 19;475(7354):110-113.

6. Tjon AS, van Gent R, Jaadar H, Martin van Hagen P, Mancham S, van der Laan LJ, te Boekhorst PA, Metselaar HJ, Kwekkeboom J. Intravenous immunoglobulin treatment in humans suppresses dendritic cell function via stimulation of IL-4 and IL-13 production. J Immunol. 2014;192(12):5625-5634.

7. Vaglio A, Moosig F, Zwerina J. Churg-Strauss syndrome: update on pathophysiology and treatment. Curr Opin Rheumatol.2012;24(1):24-30.

8. Kuo HC, Yang KD, Liang CD, Bong CN, Yu HR, Wang L, Wang CL. The relationship of eosinophilia to intravenous immunoglobulin treatment failure in Kawasaki disease. Pediatr Allergy Immunol. 2007;18(4):354-359.

9. Tseng WN, Lo MH, Guo MM, Hsieh KS, Chang WC, Kuo HC. IL-31 associated with coronary artery lesion formation in Kawasaki disease. PLoS One.2014;9(8):e105195.

10. Kuo HC, Wang CL, Liang CD, Yu HR, Huang CF, Wang L, Hwang KP, Yang KD. Association of lower eosinophilrelated $\mathrm{T}$ helper 2 (Th2) cytokines with coronary artery lesions in Kawasaki disease. Pediatr Allergy Immunol. 2009;20(3):266-272.

11. Khor CC, Davila S, Breunis WB, Lee YC, Shimizu C, Wright VJ, Yeung RS, Tan DE, Sim KS, Wang JJ, Wong TY, Pang J, Mitchell P, et al. Genome-wide association study identifies FCGR2A as a susceptibility locus for Kawasaki disease. Nat Genet. 2011;43(12):1241-1246.

12. Woon PY, Chang WC, Liang $\mathrm{CC}$, Hsu CH, Klahan $\mathrm{S}$, Huang YH, Chang WP, Kuo HC. Increased risk of atopic dermatitis in preschool children with kawasaki disease: a population-based study in taiwan. Evid Based Complement Alternat Med.2013:605123.

13. Kuo HC, Chang WC, Yang KD, Yu HR, Wang CL, Ho SC, Yang CY. Kawasaki disease and subsequent risk of allergic diseases: a population-based matched cohort study. BMC Pediatr.2013;13:38.

14. Gillis C, Gouel-Cheron A, Jonsson F, Bruhns P. Contribution of Human FcgammaRs to Disease with Evidence from Human Polymorphisms and Transgenic Animal Studies. Front Immunol. 2014;5(254).

15. Shrestha S, Wiener H, Shendre A, Kaslow RA, Wu J, Olson A, Bowles NE, Patel H, Edberg JC, Portman MA. Role of activating FcgammaR gene polymorphisms in Kawasaki disease susceptibility and intravenous immunoglobulin response. Circ Cardiovasc Genet. 2012;5(3):309-316.

16. Makowsky R, Wiener HW, Ptacek TS, Silva M, Shendre A, Edberg JC, Portman MA, Shrestha S. FcgammaR gene copy number in Kawasaki disease and intravenous immunoglobulin treatment response. Pharmacogenet Genomics. 2013;23(9):455-462.

17. Kuo HC, Chang JC, Yu HR, Wang CL, Lee CP, Huang $\mathrm{LT}$, Yang KD. Identification of an association between genomic hypomethylation of FCGR2A and susceptibility to Kawasaki disease and intravenous immunoglobulin resistance by DNA methylation array. Arthritis Rheumatol. 2015;67(3):828-836.

18. Kuo HC, Hsu YW, Wu MS, Woon PY, Wong HS, Tsai LJ, Lin RK, Klahan S, Hsieh KS, Chang WC. FCGR2A Promoter Methylation and Risks for Intravenous Immunoglobulin Treatment Responses in Kawasaki Disease. Mediators Inflamm. 2015;2015:564625.

19. Vogelpoel LT, Baeten DL, de Jong EC, den Dunnen J. Control of cytokine production by human fe gamma receptors: implications for pathogen defense and autoimmunity. Front Immunol. 2015;6:79.

20. Nagelkerke SQ, Kuijpers TW. Immunomodulation by IVIg and the Role of Fc-Gamma Receptors: Classic Mechanisms of Action after all? Front Immunol. 2014;5:674.

21. Smith KG, Clatworthy MR. FcgammaRIIB in autoimmunity and infection: evolutionary and therapeutic implications. Nat Rev Immunol. 2010;10(5):328-343.

22. Shrestha S, Wiener HW, Olson AK, Edberg JC, Bowles NE, Patel H, Portman MA. Functional FCGR2B gene variants influence intravenous immunoglobulin response in patients with Kawasaki disease. J Allergy Clin Immunol. 2011;128(3):677-680.

23. Quillien V, Lavenu A, Ducray F, Joly MO, Chinot O, Fina F, Sanson M, Carpentier C, Karayan-Tapon L, Rivet P, Entz-Werle N, Legrain M, Zalcman EL, et al. Validation 
of the high-performance of pyrosequencing for clinical MGMT testing on a cohort of glioblastoma patients from a prospective dedicated multicentric trial. Oncotarget. 2016; 7:61916-61929. doi: 10.18632/oncotarget.11322.

24. Newburger JW, Takahashi M, Gerber MA, Gewitz MH, Tani LY, Burns JC, Shulman ST, Bolger AF, Ferrieri P, Baltimore RS, Wilson WR, Baddour LM, Levison ME, et al. Diagnosis, treatment, and long-term management of Kawasaki disease: a statement for health professionals from the Committee on Rheumatic Fever, Endocarditis and Kawasaki Disease, Council on Cardiovascular Disease in the Young, American Heart Association. Circulation. 2004;110(17):2747-2771.
25. Kuo HC, Hsu YW, Wu MS, Chien SC, Liu SF, Chang WC. Intravenous immunoglobulin, pharmacogenomics, and Kawasaki disease. Journal of microbiology, immunology, and infection. 2016;49(1):1-7. 